\title{
Transition from high cardiac output to elevated vascular resistance in hypertension
}

\begin{abstract}
The early phase of hypertension (borderline hypertension) is characterized by a hyperkinetic circulation caused by excessive sympathetic drive and decreased parasympathetic inhibition to the heart. In later phases the cardiac output becomes normal, but the hypertension is still neurogenic, as demonstrated by the fact that continued pharmacologic parasympathetic, $\beta$ - and $\alpha$-adrenergic Inhibition normalizes the blood pressure. In both of these phases of the process, plasma norepinephrine values are elevated. These patients also show characteristic behavioral patterns; they are outward oriented, submissive, but experience unexpressed anger and frequently harbor hostile feelings. In late phases of hypertension the cardlac output is normal and the total peripheral resistance is elevated. This hemodynamic transition can be explained by a secondary response to elevated blood pressure. The heart becomes less responsive as a result of altered receptor responsiveness and decreased cardiac compliance, whereas the responsiveness of arterioles increases because of vascular hypertrophy, which leads to changes in the wall-to-lumen ratio. However, one observation eludes explanation: the absence of plasma norepinephrine elevation in later phases of hypertension. We propose a new conceptual framework to explain the disappearance of elevated plasma norepinephrine in the course of hypertension. The concept is based on a wide range of observations with the use of various receptor-blocking agents during neurogenic pressor responses. Invariably, the blood pressure response is preserved, but the hemodynamic pattern can be altered from a high cardiac output to high total peripheral resistance or vice versa. The "blood pressure-seeking behavior" of the central nervous system suggests that the negative feedback to the central nervous system is pressure and not flow related. If the central nervous system indeed seeks to obtain a certain pressure and maintains the high blood pressure in early phases by an elevation in cardiac output, later as structural arteriolar changes evolve and the arterioles become hyperresponsive, the same blood pressure elevation could be achieved with less sympathetic drive, and the plasma norepinephrine values return to the normal range. (AM HEART $J$ 1988;116:600.)
\end{abstract}

Stevo Julius, MD, ScD Ann Arbor, Mich.

Those of us who strongly believe in the role of the nervous system in hypertension are faced with a major dilemma. It is rather easy to demonstrate a strong neurogenic component in borderline hypertension, particularly in patients whose cardiac output is high. The evidence for the neurogenic origin of the circulatory abnormality at that stage is overwhelming. High cardiac output falls into the normal range after cardiac autonomic blockade with atropine and propranolol. ${ }^{1}$ Such patients also show characteristic behavioral patterns, which suggest that their enhanced cardiac autonomic drive may reflect a constant activation of the "defense reaction."2-4 The defense response is characterized by an increase in cardiac output and a general increase in

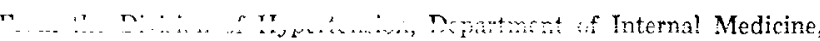
University of Michigan.

Reprint requests: Stevo Julius, MD, Division of Hypertension, Department of Internal Medicine, University of Michigan, 3918 Taubman Center, Ann Arbor, MI 48109-0356.
}

sympathetic tone. ${ }^{5}$ Finally, a subset of patients with borderline hypertension, those exhibiting high resting plasma-renin activity values, clearly have neurogenic hypertension. Their cardiac neurogenic drive is increased, plasma norepinephrine values are elevated, and the $\alpha$-adrenergic drive to the vasculature is also enhanced. ${ }^{3}$ Consequently, a complete autonomic blockade with atropine, propranolol, and regitine normalizes their blood pressure, thereby proving the prime importance of the autonomic nervous system in the genesis of blood pressure elevation.

Contrary to the good evidence for a neurogenic component in borderline hypertension, in the established phase of essential hypertension, there is very little to support a neurogenic mechanism. Instead of elevation of cardiac output, these patients show a high total peripheral resistance, their plasma norepinephrine values are not elevated, ${ }^{2}$ and there is good evidence of a structural, nonneurogenic component in the elevation of their vascular resistance. ${ }^{7,8}$ 
This apparently significant difference in the underlying mechanism of blood pressure elevation between borderline hypertension and essential hypertension is at the core of the dilemma. Are borderline hypertension and essential hypertension two different conditions, or is hypertension a process starting with borderline hypertension and later evolving into established hypertension, a process during which there is a change in the pathophysiology of the blood pressure elevation?

One can easily defend the latter position; that is, that hypertension is a process of transition from a high cardiac output to a high total peripheral resistance state. Longitudinal observations have shown normalization of the cardiac output and elevation of total peripheral resistance as patients with borderline hypertension become older., ${ }^{90}$ However, these studies have by and large failed to show a progression of blood pressure toward higher values. The void has recently been filled by $D r$. P. LundJohansen, who tenaciously followed a cohort of patients with borderline hypertension. After 10 years, the total peripheral resistance increased and cardiac output fell, but only a few developed hypertension. However, after 15 to 18 years, there was a sharp increase in subjects with classic established hypertension requiring antihypertensive treatment. ${ }^{11}$ In fact, only a few persons in the original cohort remained in the borderline hypertensive range.

The question then arises regarding whether subjects chosen for hemodynamic studies are representative of the general population. This is not an artificial academic question. We have repeatedly shown that one of the major personality characteristics of patients with borderline hypertension is submissiveness. ${ }^{12}$ Since all studies of hemodynamics describing the hyperkinetic state have been invasive, submissive persons may have selectively accepted to partake in these procedures. In turn, this may have skewed the sample so that it represents only a minor proportion of all patients with hypertension. If that were the case, the phenomenon of transition from the hyperkinetic to high-resistance hypertension would be of scientific interest but of little clinical importance. Luckily for those in our field, there is reasonable evidence that the hyperkinetic state may be a precursor of hypertension in the population at large. In the laboratory, a high cardiac output is invariably associated with a faster heart rate. If one then takes a faster heart rate to be the hallmark of the hyperkinetic state in the population at large, one gains a new perspective. A number of epidemiologic studies showed that a fast

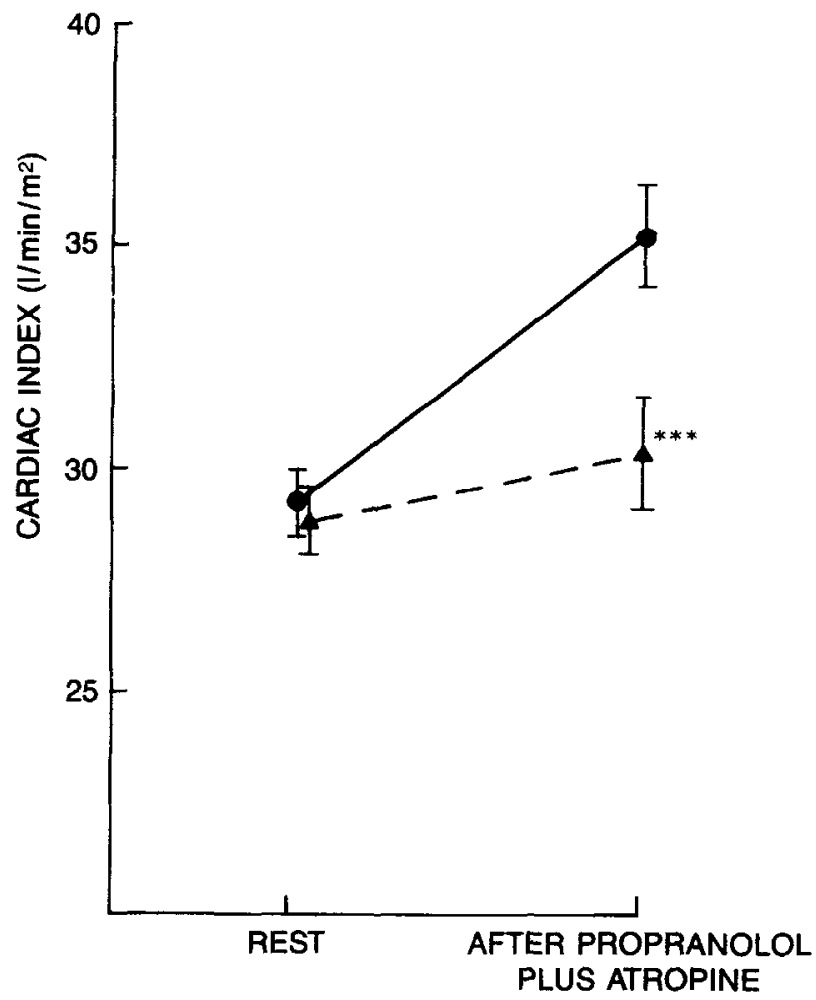

Fig. 1. Cardiac index at rest and after autonomic blockade with propranolol and atropine in 50 control subjects and 64 patients. Only patients with normal cardiac output are presented. Triangles $=$ patients; circles $=$ controls; ${ }^{* * *}=p<0.001$.

heart rate is an independent predictor of future hypertension. ${ }^{13,14}$ Particularly impressive is the study by Levy et al., ${ }^{15}$ which shows that heart rate and blood pressure are independent risks for the development of future hypertension. Persons with a fast heart rate but normal blood pressure have threefold the chance of developing hypertension than their counterparts who have both a normal blood pressure and a normal heart rate. This then suggests that in the general population, the hyperkinetic state may well precede the development of hypertension.

Since everything points to a transition from a neurogenic high cardiac output state to a nonneurogenic high-resistance state, one must try to explain the mechanisms by which the transition occurs.

Transition from high cardiac output to a high total peripheral resistance state. Many years ago Borst and Borst-de Geus ${ }^{16}$ described the autoregulatory nature of hypertension that develops after licorice ingestion. Licorice metabolizes into an aldosterone-like substance, which causes large fluid retention. In the early phase there is an elevation in cardiac output, 


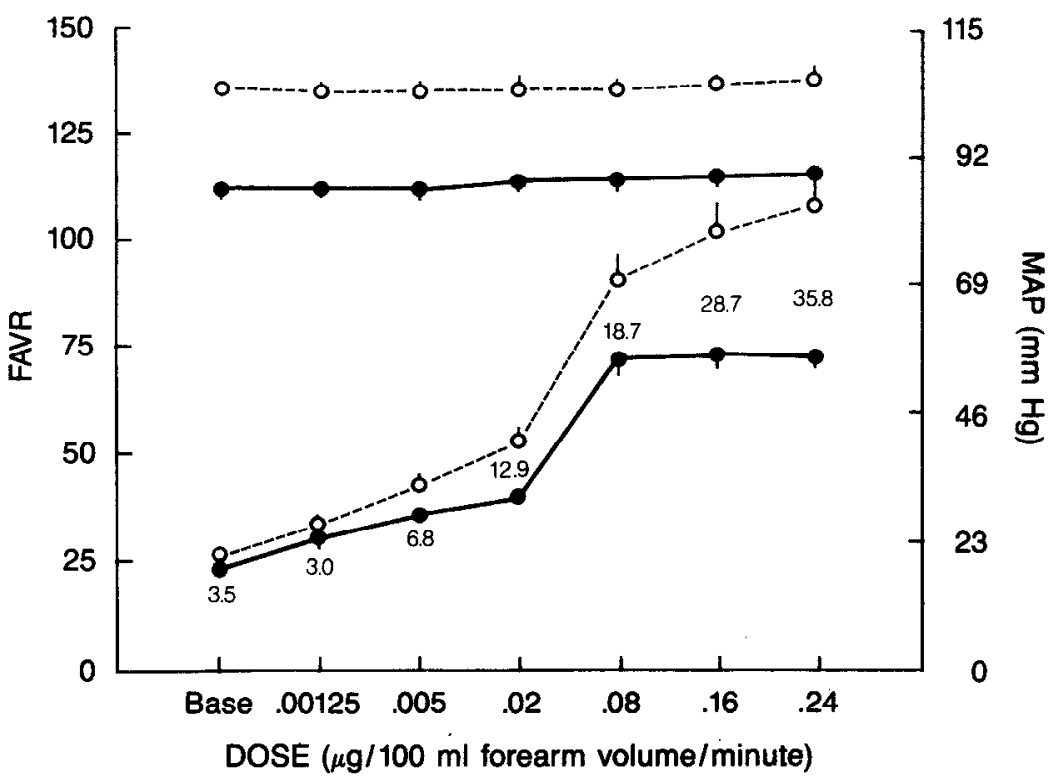

Fig. 2. The forearm vascular resistance $(F A V R)$ to norepinephrine and mean arterial pressure (MAP) $(\mathrm{mm} \mathrm{Hg})$ at baseline and in response to a sequential intra-arterial norepinephrine infusion is shown separately for hypertensives (open circles, dashed lines) and normotensives (solid circles and lines). The FAVR response curves comparing hypertensives vs normotensives were different at $p<0.001$ by analysis of variance. Although the MAP increased slightly at the higher dose, the overall MAP during the infusion was parallel in the two groups. Numbers adjacent to dose-response line represent mean differences in FAVR between the two groups.

but later the blood volume normalizes and the total peripheral resistance increases. These observations were the basis for ingenious experimentation and the development of a comprehensive theory of circulation control by Guyton and Coleman. ${ }^{17}$ The basic tenet of this theory is that elevation in cardiac output above the metabolic demands of the whole body eventually triggers an autoregulatory response of higher vascular resistance and higher blood pressure. This in turn leads to pressure diuresis, which leads to a new state of high pressure and high resistance but normal flow and volume. Autoregulation is a process whereby various tissues are capable of maintaining a constant (and optimal, in relationship to oxygen consumption) flow by adjusting the vascular resistance. An inadequate blood flow (underperfusion) will cause vasodilation, whereas overperfusion will elicit vasoconstriction. Both of these responses aim at maintaining a steady flow at the price of a variable pressure and resistance. There is good experimental evidence that such autoregulation occurs in the course of volume expansion. Despite some dissension, this is taken as evidence of total body autoregulation, that is, the ability of the whole organism to act as the sum of all local vascular beds. For the whole body, the flow is represented by the cardiac output, the resistance by total peripheral resistance, and the metabolic demands by oxygen consumption. Therefore it is tempting to explain the normalization of the cardiac output in previously hyperkinetic, borderline hypertensive persons by the process of autoregulation. Conceptually, the autoregulation will occur only if there is a state of luxurious perfusion (overperfusion); that is, the cardiac output exceeds the metabolic demands of the body. However, three independent studies ${ }^{18-20}$ have shown that together with the increased cardiac output, patients with hyperkinetic borderline hypertension also exhibit increased oxygen consumption. Since in hyperkinetic borderline hypertension both the oxygen consumption and cardiac output are elevated, the stimulus for autoregulation or overperfusion is absent; therefore such an elevation in cardiac output will not trigger an autoregulatory increase in total peripheral resistance.

Since autoregulation is not a likely explanation, what other mechanism could lead to conversion from a high cardiac output to a high resistance state? We believe that this occurs entirely through changes in structure and responsiveness of the cardiovascular system in the course of hypertension. Consequences of increased blood pressure and exaggerated sympathetic drive are such that the overall cardiac responsiveness will decrease, whereas the resistance vessel responses will be enhanced.

Lund-Johansen was the first to demonstrate that 
despite the minimal elevation in blood pressure, patients with borderline hypertension are unable to increase stroke volume during exercise to the same degree as normotensive subjects. We were later able to show a clear abnormality in resting stroke volume in borderline hypertensive patients who had normal cardiac output. ${ }^{21}$ Presumably, they had at some earlier phase a hyperkinetic circulation. These patients had faster heart rates but normal cardiac outputs; thus their resting stroke volume was decreased. That this decrease was not a simple mathematic product of increased heart rate is shown in Fig. 1. After acute autonomic blockade with intravenous propranolol and atropine, patients had significantly lower cardiac output than normotensive control subjects. The difference in cardiac output values between the two groups was entirely the result of a significant decrease in stroke volume in patients with borderline hypertension. From this we concluded that patients with normokinetic borderline hypertension probably have less compliant ventricles that fail to respond with an appropriate stroke volume to a normal preload. ${ }^{21}$ This decreased cardiac compliance is caused by secondary pressurerelated events in the heart.

In addition to a compliance-related decrease in stroke volume, the decreased chronotropic responsiveness to $\beta$-adrenergic stimulation also contributes to the decrease in cardiac output. Our studies, ${ }^{21}$ as well as those of Trimarcor et al., ${ }^{22}$ have shown subnormal heart rate responses to isoproterenol infusion in patients with normokinetic borderline hypertension. Whether this represents true receptor downregulation in response to enhanced sympathetic stimulation or some other event distal to the receptors remains to be shown.

The combination of decreased stroke volume and lesser $\beta$-adrenergic responsiveness could well explain how the previously high cardiac output returns to the normal range. However, this cannot explain the other facet of the transition to sustained hypertension, the elevation in total peripheral resistance. The movement from relatively normal to high vascular resistance can best be understood within the framework of Folkow's ${ }^{23}$ observation and his concept of vascular structural reinforcement. Arterioles respond to higher blood pressure with smooth muscle hypertrophy. Such hypertrophic muscles narrow the lumen of the blood vessels and thereby cause increased resistance to the flow. Such hypertrophic vessels with an altered wall-lumen ratio overrespond to vasoconstrictive stimuli, more than do normal vessels. The physical principles for this enhanced vasoconstriction are well explained by Folkow. $^{23}$ A practical demonstration of this

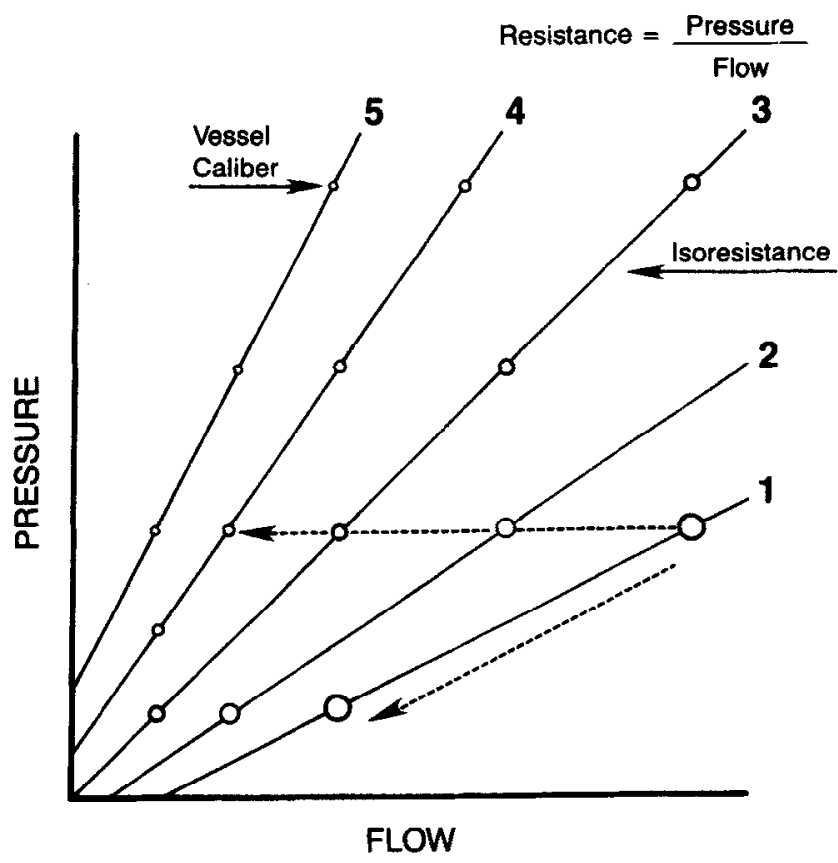

Fig. 3. The pressure-flow-resistance relationship. The diagonal lines are lines of isoresistance. Lowest diagonal line $=$ low resistance; highest $=$ high resistance. Note that the vascular caliber at low resistance is larger and that small increases of pressure cause a large increase of the flow. At the high-resistance line the vascular caliber is narrow and large increase of pressure causes only small increases of flow. Horizontal vector $=$ a decrease in flow associated with an increase in the resistance and no pressure change. Diagonal, downward-pointing vector $=$ decrease of flow with unchanged resistance and a fall in blood pressure.

enhanced responsiveness can be found in Fig. 2, which is reproduced from one of our recent studies. ${ }^{24}$

In essence, then, low-standing blood pressure and enhanced sympathetic stimulation have opposite effects on the heart and blood vessels. Structural changes will decrease the cardiac but enhance the vascular responses to sympathetic and other stimuli. This may well be the mechanism whereby the balance is shifted from a high cardiac output to a high total peripheral resistance in the course of hypertension.

Transition from a high to a normal level of sympathetic stimulation in the course of hypertension. Whereas Folkow's concept can explain the output to resistance conversion, it fails to illuminate the wellobserved fact that in the later phases of hypertension, plasma norepinephrine values are normal. That norepinephrine is elevated only in hyperkinetic borderline hypertension has been pointed out in a review by Goldstein ${ }^{6}$ and recently documented elegantly by Esler et al. ${ }^{25}$ in their studies of norepi- 


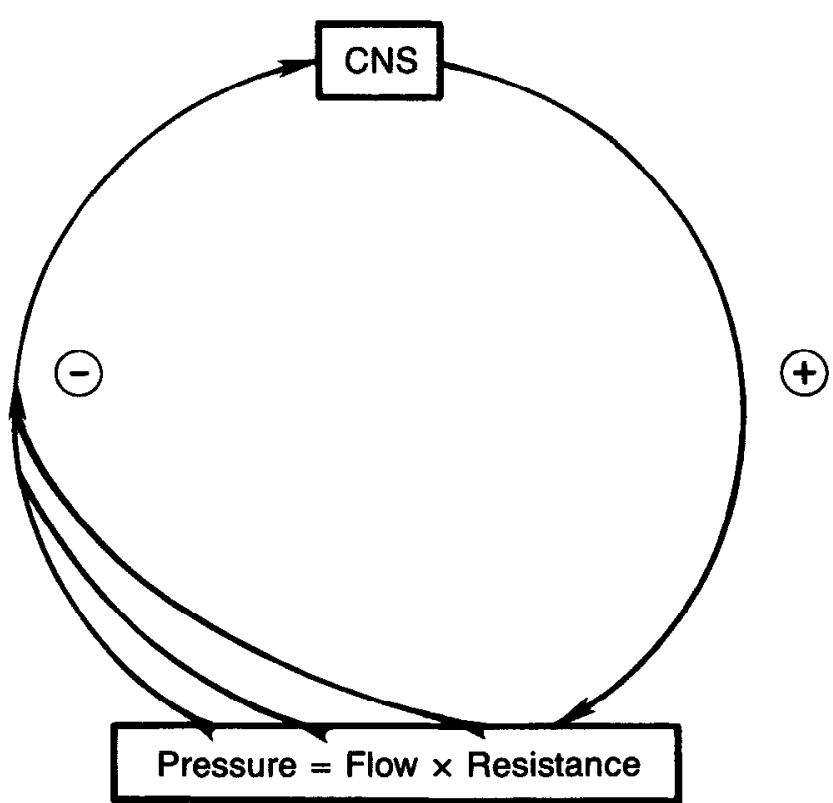

Fig. 4. The general scheme of the negative feedback from the circulation to the central nervous system (CNS).

nephrine kinetics in young and older hypertensive patients. It is easy to visualize how with the Folkow "structural reinforcement" less sympathetic firing would be needed to obtain the same vasoconstriction and blood pressure elevation. However, we know of no mechanism by which the sympathetic nervous system senses that the structural reinforcement takes place. If there is a sensing mechanism, why would the response be a decrease in sympathetic activity?

There are no direct answers to this question, but we have developed a conceptual framework that is helpful. In a recent article ${ }^{26}$ we pointed out a number of specific experimental circumstances that support the notion that the central nervous system regulates blood pressure but not flow. The concept of the "blood pressure-seeking" behavior of the central nervous system evolved from a hemodynamic analysis of acute and chronic pressor responses. The concept will now be briefly described and its relevance to the question of the disappearance of excessive sympathetic stimulation in the course of hypertension should become self-evident.

Fig. 3 presents the basic hemodynamic grid relating flow, pressure, and resistance. The flow is always a linear function of the pressure head, but the degree ol now increase tor a given ievei of pressure depends on resistance in the circuit. The resistance, in turn, depends predominantly on the cross-sectional area of the vessels. (In other systems the resistance also

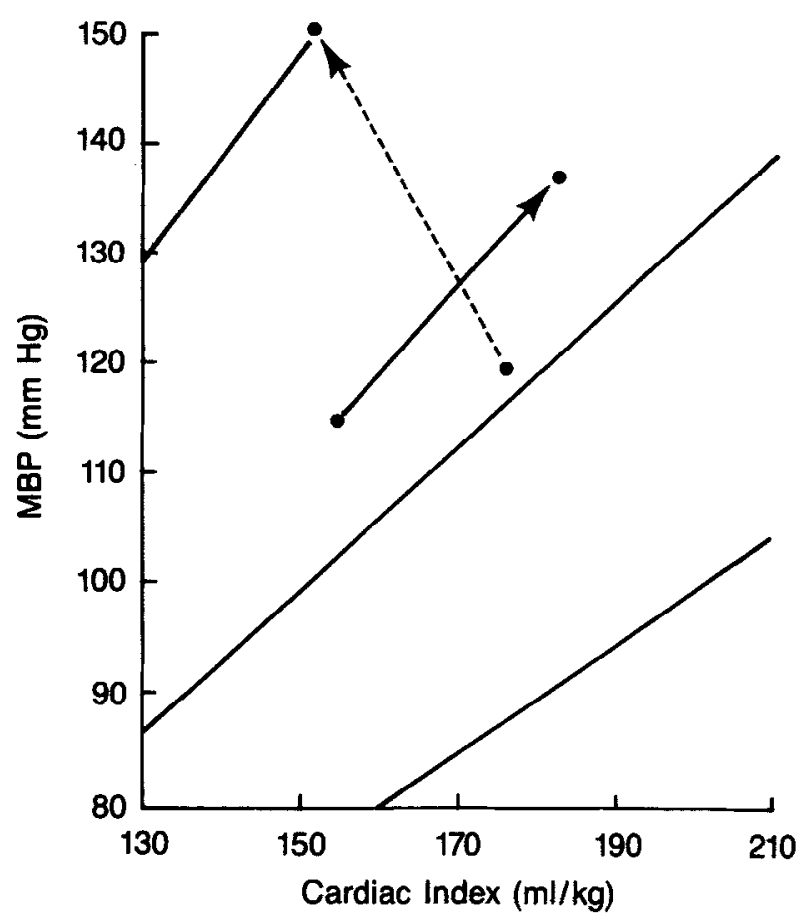

Fig. 5. Response to 60 minutes of hindquarter compression in eight chloralose-anesthetized dogs. Broken line arrow indicates the response before phenoxybenzamine. Solid line is the response to 60 minutes of compression after $1 \mathrm{mg} / \mathrm{kg}$ of intravenous phenoxybenzamine. $M B P=$ mean blood pressure. (Adapted with permission from Julius S, Sanchez R, Malayan S, et al. Sustained blood pressure elevation to lower body compression in pigs and dogs. Hypertension 1982;4:782-8.)

depends on the length of the conduit tubes, but in humans this appears to be a negligible factor.) As can be seen in the graph, when blood vessels are wide open, the resistance is low and a small increase in pressure causes a large increase in flow (right, lowest isoresistance line). When the vessels are constricted, resistance is high, and large pressure increases are required to achieve small increases in flow (left upper line). This diagram allows one to follow the direction of changes in all three components of circulation. Pressure and flow changes can be read from the corresponding axis, whereas the direction of the arrow in relation to the isoresistance lines denotes changes in resistance.

A scheme of how the central nervous system controls the circulation is shown in Fig. 4. Whenever there is a perturbance in the circulation, the central nervous sysiem acis as a negaiive ieedback, which eventually tends to limit the magnitude of the response and establishes a new equilibrium. In terms of a feedback system, the variable that is most 
highly controlled is the regulated variable. It will be shown that from three components of the circulation (resistance, flow, and pressure), the central nervous system invariably chooses to maintain certain pressure while allowing large variations in cardiac output (flow) and vascular resistance. We call this phenomenon the "blood pressure-seeking property of the central nervous system." Fig. 5 illustrates one such instance in which the central nervous system appears to seek a pressure, regardless of the state of vascular resistance or cardiac output. When hindquarters of dogs are exposed to external compression through a pressure suit, this elicits a strong and long-lasting neurogenic increase in the blood pressure. ${ }^{27,}{ }^{28}$ The usual hemodynamics of this response is an increase in vascular resistance (arrow). When these dogs were pretreated with dibenzyline to abolish the $\alpha$-adrenergic drive to the resistance vessels, the blood pressure response was essentially the same, but now all the increase was the result of an elevation in the cardiac output. This plasticity, where the underlying hemodynamic components are changed but the blood pressure response is preserved, has been demonstrated under a wide range of circumstances. Reactions to mental arithmetic, quiet talking, and isometric exercise are reflex responses in which normal hemodynamics consist of an increase in blood pressure associated with an elevation in cardiac output. $\beta$-Adrenergic blockade $^{29,30}$ does not alter the magnitude of these blood pressure increases, but uniformly changes them from a high output to a high-resistance response. Obviously, when the ability to increase the cardiac output is abolished by $\beta$-adrenergic blockade, the balance of the autonomic discharge to the blood vessels changes, causing vasoconstriction to preserve the required blood pressure response. The situation is similar after myocardial infarction. Patients with poor myocardial performance respond to isometric exercise with a normal increase in blood pressure, but achieve this increase through high resistance without changes in cardiac output. ${ }^{31}$ The conversion of primary cardiac output-mediated blood pressure response to a resistance blood pressure elevation has also been demonstrated after ganglion stellate stimulation in dogs ${ }^{32}$ Further evidence of the preserved blood pressure regulation has been shown in subjects treated with antihypertensive drugs. $\beta$-Blocking agents alter the cardiac output elevation to an elevation in vascular resistance, ${ }^{33}$ whereas a calcium antagonist alters the response to a fall in vascular resistance with excessive increase in cardiac output. ${ }^{34}$ However, both drugs do not affect the magnitude of the blood pressure response.
In a few instances neurogenic blood pressure increases are primarily mediated by an increase in vascular resistance (cold pressor and response to noise). The effect of antihypertensive treatment on the response to noise has been investigated. It was again shown that vasodilators will not affect the blood pressure response, but they convert the underlying hemodynamics from a resistance to a cardiac output-mediated response. ${ }^{35}$

From this one can conclude that for the central nervous system, pressure is the primary regulated variable; therefore feedback to the central nervous system must be related to the achieved pressure. To express it in vernacular terms, the central nervous system "desires" a certain pressure commensurate to a given situation and switches the autonomic tone from one to the other organ until it achieves the pressure. The mechanism by which the central nervous system senses the achieved pressure is not known. It is conceivable that some higher centers exert inhibitory influences on the afferent input from the peripheral mechanoreceptors. Alternatively, one cannot rule out the possibility that the central nervous system uses another yet unexplored set of pressure sensors.

If one accepts the notion that in subserving the circulation, the central nervous system primarily regulates blood pressure and has sensors to assess whether the desired pressure has been achieved, it takes only a small leap of imagination to postulate what could happen in the course of the natural history of hypertension. Were this center for some (possibly behavior-related) reason reset to function at a slightly higher set point in hypertension, at the beginning the higher "desired" pressure could only be achieved by an enhanced autonomic cardiovascular drive. In a later phase when the arteriolar responsiveness increases as a result of structural reinforcement, less sympathetic drive would be needed to achieve the "desired" pressure. The pressure sensor may then decrease the sympathetic drive. In this new state, from the standpoint of sympathetic firing rates, mild blood pressure elevation may be achieved more economically, and the plasma norepinephrine, a rough indicator of the central sympathetic discharge, may indeed return to a normal range. What started as neurogenic hypertension may now, on the surface, appear to be unrelated to any sympathetic overactivity.

\footnotetext{
REFERENCES

1. Julius S, Pascual AV, London R. Role of parasympathetic inhibition in the hyperkinetic type of borderline hypertension. Circulation 1971;44:413-8.

2. Harburg E, Julius S, McGinn NF, McLeod J, Hoobler SW.
} 
Personality traits and behavioral patterns associated with systolic blood pressure levels in college males. J Chronic Dis 1964;17:405-14.

3. Esler M, Julius S, Randall O, DeQuattro V, Zweifler A. High-renin essential hypertension: adrenergic cardiovascular correlates. Clin Sci 1976;51:181s-4s.

4. Schneider RH, Egan BM, Johnson EH, Drobny H, Julius S. Anger and anxiety in borderline hypertension. Psychosom Med 1986;48:242-8.

5. Brod J. Hemodynamic basis of acute pressor reactions and hypertension. Br Heart J 1963;25:227-45.

6. Goldstein DS. Plasma catecholamines and essential hypertension. An analytical review. Hypertension 1983;5:86-99.

7. Sivertsson R. The hemodynamic importance of structural vascular changes in essential hypertension. Acta Physiol Scand 1970;79(suppl 343):3-56.

8. Conway J. A vascular abnormality in hypertension. A study of blood flow in the forearm. Circulation 1963;27:520-9.

9. Eich RH, Cuddy RP, Smulyan H, Lyons RH. Hemodynamics in Iabile hypertension: a follow-up study. Circulation 1966; 34:299-307.

10. Safar ME, Weiss YA, Levenson JA, London GM, Milliez PL. Hemodynamic study of 85 patients with borderline hypertension. Am J Cardiol 1973;31:315-9.

11. Lund-Johansen $P$. Hemodynamic alterations in early essential hypertension: Recent advances. In: Gross F, Strasser T, eds. Mild hypertension: recent advances. New York: Raven Press, 1983:237-49.

12. Julius $\mathrm{S}$. The psychophysiology of borderline hypertension. In: Weiner H, Hofer MA, Stunkard AJ, eds. Brain, behavior, and bodily disease. New York: Raven Press, 1981:293-303.

13. Paffenbarger RS, Thorne MC, Wing AL. Chronic disease in former college students-VIII. Characteristics in youth predisposing to hypertension in later years. Am J Epidemiol 1968;88:25-32

14. Stamler J, Berkson DM, Dyer A, et al. Relationship of multiple variables to blood pressure: findings from four Chicago epidemiologic studies. In: Paul $\mathbf{O}$, ed. Epidemiology and control of hypertension. Miami: Symposia Specialists, 1975:307-52.

15. Levy RL, White PD, Stroud WD, Hillman CC. Transient tachycardia: prognostic significance alone and in association with transient hypertension. JAMA 1945;129:585-8.

16. Borst JGG, Borst-de Geus A. Hypertension explained by Starling's theory of circulatory homeostasis. Lancet 1963; 1:677-82.

17. Guyton AC, Coleman TG. Quantitative analysis of the pathophysiology of hypertension. Circ Res 1967;24-25(suppl I):I19.

18. Lund-Johansen P. Hemodynamics in early essential hypertension. Acta Med Scand 1967;482(suppl):1-105.

19. Sannerstedt R. Hemodynamic response to exercise in patients with arterial hypertension. Acta Med Scand 1966;458(suppl):1-83.
20. Julius $\mathrm{S}$, Conway J. Hemodynamic studies in patients with borderline blood pressure elevation. Circulation 1968;38:282 8.

21. Julius S, Randall OS, Esler MD, Kashima T, Ellis CN Bennett J. Altered cardiac responsiveness and regulation in the normal cardiac output type of borderline hypertension. Circ Res 1975;36-37(suppl I):I199-I207.

22. Trimarco B, Volpe M, Ricciardelli B, Galva MA, Petracca R Condorelli M. Studies of the mechanisms underlying impairment of beta-adrenoceptor mediated effects in human hypertension. Hypertension 1983;5:584-90.

23. Folkow B. Physiological aspects of primary hypertension. Physiol Rev 1982;62:347-503.

24. Egan B, Panis R, Hinderliter A, Schork N, Julius S. Mechanism of increased alpha-adrenergic vasoconstriction in human essential hypertension. J Clin Invest 1987;80:812-7.

25. Esler M, Jennings G, Biviano B, Lambert G, Hasking G. Mechanism of elevated plasma noradrenaline in the course of essential hypertension. J Cardiovasc Pharmacol 1986;8(suppl 5):S39-S43.

26. Julius $\mathbf{S}$. The blood pressure seeking properties of the central nervous system. J Hypertens 1988;6:177-85.

27. Julius S, Sanchez R, Malayan S, et al. Sustained blood pressure elevation to lower body compression in pigs and dogs. Hypertension 1982;4:782-8.

28. Osterziel KJ, Julius S, Brant D. Blood pressure elevation during hindquarter compression in dogs is neurogenic. J Hypertens 1984;4:411-7.

29. Ulrych $M$. Changes of general hemodynamics during stressful mental arithmetic and nonstressing quiet conversation and modification of the latter by beta-adrenergic blockade. Clin Sci 1969;36:453-61.

30. Donald KW, Lind AR, McNicol GW, Humphreys PW, Taylor SH, Staunton HP. Cardiovascular responses to sustained (static) contractions. Circ Res 1967;20-21(suppl I):I15-I30.

31. Baccelli G, Valentini R, Gregorini L, Cellina G, Mancia G, Ludbrook J, Zanchetti A. Hemodynamic effects of isometric handgrip exercise in patients convalescent from myocardial infarction. Clin Exp Pharmacol Physiol 1978;5:607-15.

32. Liard JF, Tarazi RC, Ferrario CM, Manger WM. Hemodynamic and humoral characteristics of hypertension induced by prolonged stellate ganglion stimulation in conscious dogs. Circ Res 1975;36:455-64

33. Andren L, Hansson L. Circulatory effects of stress in essential hypertension. Acta Med Scand 1980;646:69-72.

34. Schmieder RE, Rüddel $H$, Neus H, Messerli FH, Von Eiff AW. Disparate hemodynamic responses to mental challenge after antihypertensive therapy with beta blockers and calcium entry blockers. Am J Med 1987;82:11-6.

35. Andren L, Hansson L, Eggertsen R, Hedner T, Karlbert BE. Circulatory effects of noise. Acta Med Scand 1983;213:31-5. 